\title{
Comparison of peak oxygen consumption response to aquatic and robotic therapy in individuals with chronic motor incomplete spinal cord injury: a randomized controlled trial
}

\author{
Peter H. Gorman $\mathbb{1}^{1,2,3} \cdot$ William Scott ${ }^{2}$ - Leslie VanHiel ${ }^{4,5} \cdot$ Keith E. Tansey ${ }^{4,6} \cdot$ W. Mark Sweatman ${ }^{4}$. \\ Paula Richley Geigle ${ }^{1,2}$
}

Received: 1 May 2018 / Revised: 14 December 2018 / Accepted: 2 January 2019 / Published online: 18 January 2019

(c) International Spinal Cord Society 2019

\begin{abstract}
Study design: Randomized dual center controlled clinical trial.

Objective: To determine and compare the cardiorespiratory impact of 3 months of aquatic and robotic therapy for individuals with chronic motor incomplete spinal cord injury (CMISCI).

Settings: Two rehabilitation specialty hospitals.

Methods: Thirty-one individuals with CMISCI with neurological level between C2-T12 at least 1 year post injury were randomized to either aquatic or robotic treadmill therapy for 36 sessions. Customized sessions lasted 40-45 min at 65-75\% heart rate reserve intensity with peak oxygen consumption (peak $\mathrm{VO}_{2}$ ) measured during arm ergometry at baseline and post intervention. Additional peak robotic treadmill $\mathrm{VO}_{2}$ assessments were obtained before and after training for participants randomized to robotic intervention.

Results: Peak $\mathrm{VO}_{2}$ measured with arm ergometry was not significantly different with either aquatic intervention $(8.1 \%, p=$ $0.14, n=15)$ or robotic intervention $(-0.7 \%, p=0.31, n=17)$. Peak $\mathrm{VO}_{2}$ measured with robotic treadmill ergometry demonstrated a statistical improvement $(14.7 \%, p=0.03, n=17$, two-tailed $t$-test) across the robotic intervention. Comparison between the two interventions demonstrated a trend favoring aquatic therapy for improving arm ergometry peak $\mathrm{VO}_{2}$ (ANOVA, $p=0.063$ ).

Conclusions: Neither 3-month exercise interventions statistically improved arm cycle ergometry peak $\mathrm{VO}_{2}$, our cardiorespiratory surrogate marker, although percent improvement was greater in the aquatic exercise condition. Robotic ergometry peak $\mathrm{VO}_{2}$ did improve for the robotic intervention, confirming previous work. These results suggest that either intervention may hold utility in improving cardiorespiratory fitness in CMISCI, but peak $\mathrm{VO}_{2}$ measurement technique appears critical in detecting effects.

Sponsorship: DOD CDMRP SCI Research Program Clinical Trial Award SC090147, FY 2009. This study is registered under ClinicalTrials.gov Identifier: NCT01407354.
\end{abstract}

Supplementary information The online version of this article (https:// doi.org/10.1038/s41393-019-0239-7) contains supplementary material, which is available to authorized users. \footnotetext{
\begin{tabular}{ll}
\hline & $\begin{array}{l}\text { Peter H. Gorman } \\
\text { pgorman @umm.edu }\end{array}$ \\
$1 \quad$ & University of Maryland School of Medicine, Baltimore, MD, USA \\
2 & $\begin{array}{l}\text { University of Maryland Rehabilitation and Orthopaedic Institute, } \\
\text { Baltimore, MD, USA }\end{array}$
\end{tabular} \begin{tabular}{ll}
\hline & $\begin{array}{l}\text { Peter H. Gorman } \\
\text { pgorman @umm.edu }\end{array}$ \\
$1 \quad$ & University of Maryland School of Medicine, Baltimore, MD, USA \\
2 & $\begin{array}{l}\text { University of Maryland Rehabilitation and Orthopaedic Institute, } \\
\text { Baltimore, MD, USA }\end{array}$
\end{tabular} \begin{tabular}{ll}
\hline & $\begin{array}{l}\text { Peter H. Gorman } \\
\text { pgorman @umm.edu }\end{array}$ \\
$1 \quad$ & University of Maryland School of Medicine, Baltimore, MD, USA \\
2 & $\begin{array}{l}\text { University of Maryland Rehabilitation and Orthopaedic Institute, } \\
\text { Baltimore, MD, USA }\end{array}$
\end{tabular} \begin{tabular}{ll}
\hline & $\begin{array}{l}\text { Peter H. Gorman } \\
\text { pgorman@umm.edu }\end{array}$ \\
1 & University of Maryland School of Medicine, Baltimore, MD, USA \\
2 & $\begin{array}{l}\text { University of Maryland Rehabilitation and Orthopaedic Institute, } \\
\text { Baltimore, MD, USA }\end{array}$
\end{tabular} \begin{tabular}{ll}
\hline & $\begin{array}{l}\text { Peter H. Gorman } \\
\text { pgorman @umm.edu }\end{array}$ \\
$1 \quad$ & University of Maryland School of Medicine, Baltimore, MD, USA \\
2 & $\begin{array}{l}\text { University of Maryland Rehabilitation and Orthopaedic Institute, } \\
\text { Baltimore, MD, USA }\end{array}$
\end{tabular}
}

\section{Introduction}

Aerobic cardiorespiratory fitness is comparatively low in the spinal cord injury (SCI) population, with approximately $25 \%$ of otherwise healthy young individuals with SCI failing to achieve oxygen consumption levels sufficient to

\footnotetext{
VA Maryland Healthcare System, Baltimore, MD, USA

Shepherd Center, Atlanta, GA, USA

5 Ekso Bionics, Inc., Richmond, CA, USA

6 Methodist Rehabilitation Center/University of Mississippi Medical Center/Jackson VA Medical Center, Jackson, MS, USA
} 
perform many essential activities of daily living (ADLs) [1]. Interventions to effectively increase cardiorespiratory endurance and exercise capacity include arm ergometry [2-4], functional electrical stimulation [5], and robotically assisted treadmill training [6, 7]. Six months, three times weekly, of body weight supported treadmill training produced both glucose and insulin reduction in people with chronic incomplete SCI [8]. However, a Cochrane review of the effect of exercise on carbohydrate and lipid metabolism disorders in people with chronic SCI concluded insufficient evidence exists to determine whether exercise can improve carbohydrate and lipid metabolism [9].

Body weight supported locomotor training with or without robotic assistance has been shown to improve walking and cardiorespiratory fitness for people with chronic motor incomplete spinal cord injury (CMISCI) [10-15]. Jack et al. [13] reported improvement in peak $\mathrm{VO}_{2}$ uptake on two participants who engaged in body weight supported treadmill training without robotic assistance three times per week for 20 weeks. Kressler et al. [14] performed an extensive single site comparison study of four different approaches to body weight supported locomotor training: (1) manual assistance, (2) transcutaneous electrical stimulation, (3) robotically assisted training, or (4) over ground training with electrical stimulation. The robotically assisted training group failed to display a change in peak $\mathrm{VO}_{2}$, whereas individuals in the other three groups demonstrated improved peak $\mathrm{VO}_{2}$ responses after the intervention. The passive robotic training paradigm employed in this study utilizing $100 \%$ guidance force to provide maximal assistance throughout the step cycle may have contributed to no robotic cardiorespiratory change [16].

We previously reported improvement in peak $\mathrm{VO}_{2}$ after a 3-month, three times per week coached robotic-assisted body weight supported treadmill training (RABWSTT) [6]. This prospective randomized single center controlled clinical trial involved 18 individuals with CMISCI randomized to progressive robotic therapy or a home stretching control arm. Outcome measures included peak $\mathrm{VO}_{2}$ measured with both robotic-assisted treadmill walking ergometry and arm ergometry at baseline, 6 weeks, and 3 months. Peak $\mathrm{VO}_{2}$ measured with robotic treadmill ergometry across the robotic therapy intervention showed a statistically significant improvement of $12.3 \%$ from baseline to 3 months $(p=0.02)$ compared to the stretching control group nonsignificant improvement of $3.9 \%(p=0.37)$. Peak $\mathrm{VO}_{2}$ measured with arm ergometry in the robotic therapy group increased, albeit not significantly, by $8.5 \%(p=0.25)$.

We also demonstrated high test-retest reliability of peak $\mathrm{VO}_{2}$ measurement during robotic treadmill walking $(r=$ $0.96, p=0.01)$ and arm ergometry $(r=0.95, p=0.01)$ in this population [17], as well as a high correlation $(r=0.87$, $p=0.01)$ between arm cycle ergometry and robotic treadmill peak $\mathrm{VO}_{2}$ testing. This suggests both techniques assess cardiorespiratory fitness, although different peak $\mathrm{VO}_{2}$ outcomes are expected when using only upper extremities, or upper and lower extremities and trunk.

Aquatic therapy is a rehabilitation technique utilized frequently to improve function for individuals with CMISCI [18, 19]. Limited empirical evidence exists, however. Clinically, the aquatic environment facilitates both gait and cardiorespiratory functional gains. Buoyancy provides natural body weight support; hydrostatic pressure activates sensory receptors, thereby providing increased proprioceptive feedback; viscosity increases resistance to the musculoskeletal and cardiorespiratory systems. Stevens et al. [20] recently reported improved leg strength, balance, walking speeds, distance walked over 6 min, and daily step activity in 11 volunteers with incomplete SCI who participated in an 8-week, three times per week underwater treadmill training program. They did not address aerobic capacity, however.

Given this background, we proposed a randomized clinical trial to assess two rehabilitation techniques for individuals with CMISCI. Our a priori hypotheses anticipated both conditions would improve cardiorespiratory fitness but aquatic therapy (AT) would demonstrate a ten percent greater improvement than robotic-assisted body weight supported treadmill training (RT). We based this on the idea buoyancy facilitates more innervated motor units to contract due to the relative reduction of gravity and hydrostatic pressure assists with blood flow return. Both hydrodynamic principles facilitate a greater work load for individuals with SCI than a land-based exercise program, even body weight supported activities. We assessed the impact of 36 sessions of AT and RT on cardiorespiratory fitness for individuals with CMISCI. This paper represents one component of our overall study examining aquatic and robotic treadmill therapy effects prior to crossover to the alternate intervention upon cardiorespiratory fitness.

\section{Methods}

The appropriate institutional review boards (IRBs) as well as the Department of Defense IRB approved this protocol. This trial involved collaborating sites at two rehabilitation specialty hospitals located in different United States cities. Weekly communication occurred between intervention practitioners across both sites, and monthly teleconferences occurred between the principal investigators and practitioners to insure adherence to the protocol and offer optimal programming for participants. During data collection lead investigators visited each others' sites on two occasions and conducted aquatic and robotic training sessions. Additionally, throughout the study weekly phone calls and emails occurred across the entire study period to address 
procedural consistency, and the research team met annually at professional conferences to discuss study logistics.

\section{Statement of compliance}

We certify that all applicable institutional and governmental regulations concerning the ethical use of human volunteers were followed during this research. This trial was registered under Clinicaltrials.gov identifier NCT01407354.

\section{Participants}

Individuals with CMISCI were recruited as a convenience sample from outpatient SCI clinics and through available clinical databases at both centers. The recruitment of individuals for this study occurred over three and a half years. Participants eligible for the study were between the ages of 18 and 65 , with a spinal cord injury of at least 12 months, including levels $\mathrm{C} 2$ to $\mathrm{T} 12$, and classified as an International Standards for Neurological Classification of Spinal Cord Injury (ISNCSCI) impairment scale of C or D, who could tolerate being upright in a standing frame for at least $30 \mathrm{~min}$. We excluded individuals with a history of long bone fracture, active symptomatic cardiovascular disease, concurrent lower motor neuron injury as determined by physical examination (areflexia), other medical co-morbidities precluding safe participation, or active participation in physical therapy for 3 months prior to enrollment.

Participant screening included a general history and physical examination (done by a spinal cord injury certified physician at each site), an ISNCSCI examination (done by a spinal cord injury specialty trained physical therapist or physician), a resting electrocardiogram, and baseline blood tests including a complete blood count and metabolic profile to screen for occult infection, anemia, renal or liver disease, or uncontrolled diabetes. A $30 \mathrm{~min}$ standing frame challenge was performed by a physical therapist. Information collected included The Lower Extremity Motor Score (LEMS), which is derived from five specific bilateral leg muscle scores included in the ISNCSCI examination completion; and the Upper Extremity Motor Score (UEMS) obtained in a similar manner; The Walking Index for Spinal Cord Injury (WISCI-III), which records the physical assistance needed, and devices required, for walking following spinal cord injury. In addition, basic demographic information (age, sex, age at time of injury, time since injury) was collected on all individuals. Participants meeting eligibility criteria and who signed the IRB approved consent forms were block randomized by site using a computer-generated algorithm to treatment with either robotic or aquatic therapy for 36 sessions followed by repeat peak $\mathrm{VO}_{2}$ testing. A statistician not involved in the study generated the randomization. The randomization was implemented after all baseline testing was completed and was performed by a research coordinator uninvolved in the study execution. For individual subjects, the screening, randomization process, and baseline physiologic testing (see below) took approximately 2 weeks.

\section{Baseline and outcome physiologic testing}

Testing was performed by appropriately trained personnel at each site. This included an exercise physiologist and physical therapist at one site, and a blinded physical therapist at the second site. The arm cycle ergometry was performed in a clinical laboratory research setting at each site. The robotic-assisted ergometry testing was performed in clinical treatment space at one facility and in dedicated research space where the respective Lokomat ${ }^{\circledR}$ devices were located.

\section{Peak oxygen consumption measurement}

Peak aerobic capacity was assessed under two conditions: (1) arm ergometry and (2) robotic treadmill walking. A COSMED Quark Cardiopulmonary Metabolic Cart (CPET) measured oxygen consumption during the peak aerobic tests (arm ergometry and robotic treadmill walking) with the unit calibrated per manufacturer guidelines before each test. A Hans Rudolph half mask with a flow meter attachment was positioned over the participant's nose and mouth region, permitting the collection of continuous air flow and gas concentrations. This configuration connected to the metabolic cart and a computer software program integrating the flow and gas data to calculate oxygen consumption values. We determined peak oxygen consumption by averaging the highest values observed from three consecutive 10-s sampling periods.

\section{Arm ergometry peak $\mathrm{VO}_{2}$ test}

We conducted the arm ergometry test with a Monark upper extremity arm cycle ergometer with participants positioned in front of the device in a standard chair or personal wheelchair. The ergometer height aligned the axis of rotation with the shoulder joint with seating adjustments made to bring the pelvis in a neutral position. Participants sat quietly for $5 \mathrm{~min}$ before performing a 3-min warm-up phase at zero watts (no resistance) with the pedal cadence at $50 \mathrm{rpm}$. Work rates increased by 5 watts every minute after the warm-up phase with pedal cadence remaining constant at $50 \mathrm{rpm}$. The session terminated at volitional fatigue or if the participant failed to maintain the pedal cadence at $50 \mathrm{rpm}$ at any given work rate. 


\section{Robotic-assisted body weight supported peak $\mathrm{VO}_{2}$ test}

We used a robotic device, the Lokomat ${ }^{\circledR}$ Robotic Treadmill training device (Hocoma, Inc.), with a computer interface for the robotic treadmill walking ergometry test. All participants were measured and configured in the device according to manufacturer guidelines. Participants were positioned safely with as much body weight as possible and were instructed to limit movement and communication for a 5 -min period prior to the initiation of the treadmill test to collect baseline $\mathrm{VO}_{2}$ and heart rate values. Next, participants performed a 3-min warm-up phase at his or her optimal treadmill speed and body weight support (BWS). We determined the optimal work rate (speed and BWS) during a 20-min acclimation training session, which determined treadmill speed and BWS for an optimal gait pattern. We changed this work rate each minute, first by decreasing BWS by $10 \%$ increments, next by increasing speed by 0.2 kilometers/hour, and then by decreasing guidance force by $10 \%$ increments. Participants walked until they reached volitional fatigue or failed to maintain a safe gait pattern (e.g., without tripping or stumbling).

The testers that performed these peak $\mathrm{VO}_{2}$ tests at the second site were trained in the standardized implementation of these tests by an exercise physiologist (author WS) with previous expertize in these types of studies, who performed all of the testing at the first site. Because of the relatively small size of the research teams at one site, blinding of testers was not practicable. The second site was able to employ an external therapist for testing other than robotic peak $\mathrm{VO}_{2}$.

\section{Interventions}

The interventions were performed by a team of skilled physical or occupational therapists at each site. During each training session for both groups, heart rate was assessed by palpation and with a heart rate monitor to guide tasks to stimulate the desired exercise intensity $(65-75 \%$ heart rate reserve (HRR)) as determined during the baseline peak $\mathrm{VO}_{2}$ test. The Borg scale (6-20) was also administered to all participants to obtain subjective feedback regarding the exercise intensity and was used as an additional tool to adjust exercise intensity during and between sessions [21].

\section{Robotic therapy (RT) intervention}

Participants reported to the rehabilitation facility up to three days per week during the robotic training intervention using Lokomat ${ }^{\circledR}$. An acclimation session was performed to determine the robot manufacturer guideline anatomical settings, and optimal treadmill speed, body weight support, and guidance force settings before conducting robotic ergometry testing and the intervention. To allow endurance and skin tolerance to build, the first robotic session duration was $20 \mathrm{~min}$, and then increased by 5 -min in subsequent visits until the exercise duration reached $45 \mathrm{~min}$. During each session participants performed a 5-min warm-up, followed by customized, coached gait training tasks, and a 5-min cool-down. During each training session, the Polar heart rate monitor, as well as palpated heart rate, guided clinician application of treadmill speed, body weight support, guidance force adjustments, and coaching to stimulate the desired exercise intensity. The overall protocol for adjustment of the robotic treadmill parameters is depicted in Figure1. Stimulus intensity was adjusted in an individualized way in order to achieve 65 to $75 \%$ HRR.

\section{Aquatic therapy (AT) intervention}

Aquatic therapy sessions consisted of up to three, 45-min sessions per week. Training started conservatively at $50 \%$ of HRR and advanced to $65-75 \%$ HRR (measured also by a Polar heart rate monitor and palpation) as determined by the adjusted-for-aquatic-immersion Karvonen formula [22]. The initial session duration was $20 \mathrm{~min}$, with each subsequent session increased by $5-7 \mathrm{~min}$ until $45 \mathrm{~min}$ was reached. Multiple factors influence heart rate during aquatic activity, and the aquatic-immersion-adjusted Karvonen heart rate addressed these components such as depth of immersion and water temperature. Each session consisted of 5-min warm-up and cool-down periods, and equal training time of mobility, cardiorespiratory, and strengthening components with rest periods of 1- to 3-min as needed. Participant ability and motor function directed our customized and individualized exercise routines, but followed the protocol guidelines (Table 1). Water positioning (standing, sitting, horizontal, depth), floatation and resistance devices used, and heart rate and Borg exertion scale reports were assessed and recorded daily. Therapists encouraged each participant to work at $65-75 \%$ intensity level throughout each aquatic exercise session.

\section{Statistical analysis}

Prior to initiation of the study, a sample size analysis was performed. There was no prior data available on RT or AT in individuals with CMISCI on which to base a power calculation. There was however, prior work on cardiorespiratory fitness in a spinal cord injured cohort using aerobic wheelchair propulsion as the exercise intervention. Gass et al. [23] in this early work, physically trained seven spinal cord injured individuals for 7 weeks, five times per week (35 sessions) using wheelchair pushing on a treadmill and measured $\mathrm{VO}_{2 \max }$ before and after the intervention. They found a $35 \%$ increase in $\mathrm{VO}_{2 \max }$. This study involved 
Fig. 1 Robotically Assisted Treadmill Training Protocol using Lokomat ${ }^{\oplus}$ : First session and start of each subsequent session

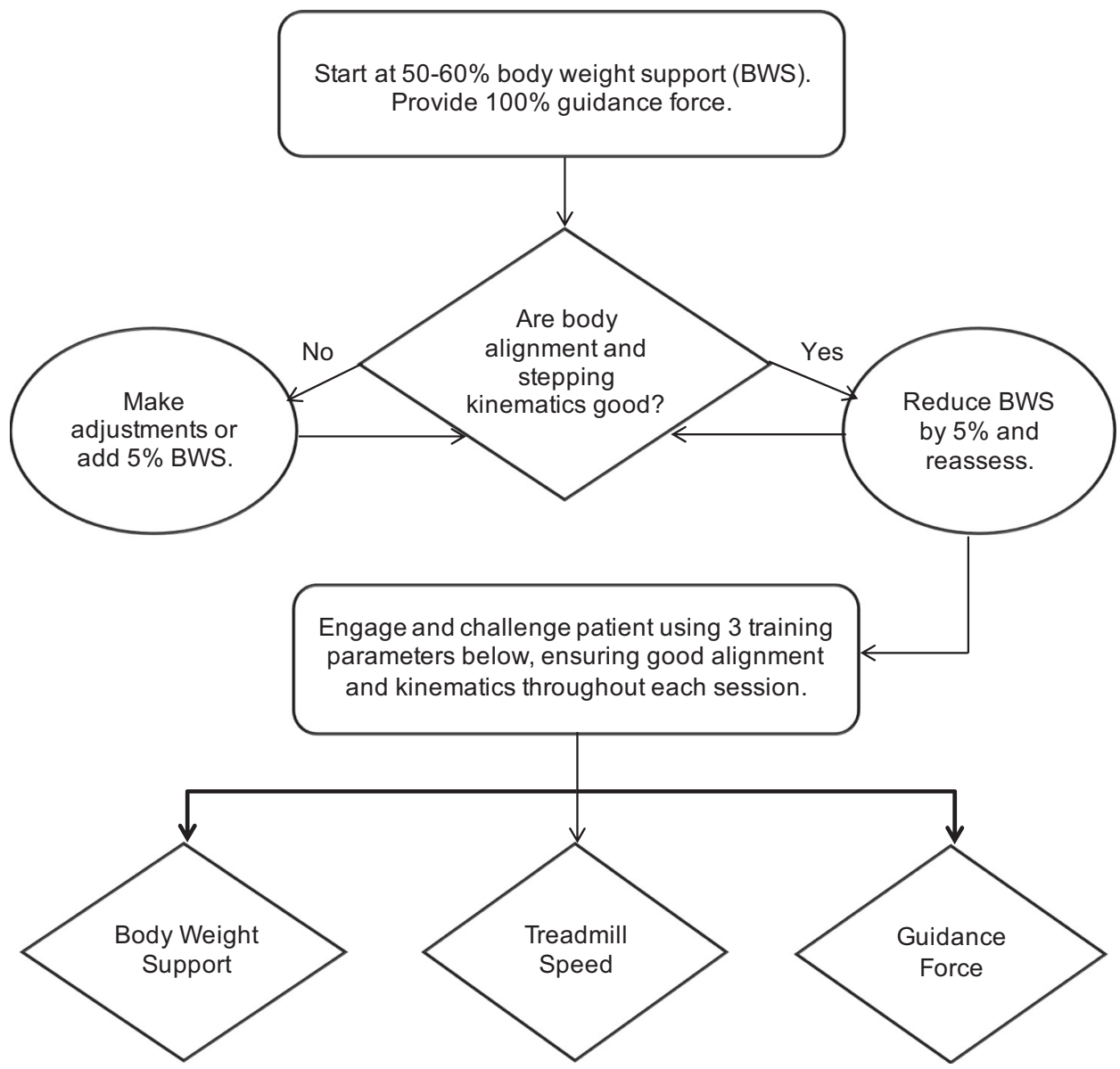

a similar intensity of exercise experience as what had been proposed for the current work. Based on that prior result, with an alpha of 0.05 and power of $0.8,28$ total participants were estimated to be needed for this study.

We used Microsoft Excel to manage data throughout the trial, checking for data completeness, regularity, and coding. All statistical analyses utilized SPSS version 22. Descriptive statistics, $t$-tests and ANOVA were performed. Descriptive statistics detailed demographics within the sample and independent means. Means and standard deviations were generated for the primary outcome measure (peak $\mathrm{VO}_{2}$ ). Paired sampled $t$-tests were performed to determine if peak $\mathrm{VO}_{2}$ changes occurred in groups pre- and post-intervention. Significance was set a priori with alpha $=0.05$. A modified intention to treat analysis was performed in which all participants with available data were analyzed according to their group allocations.

\section{Results}

We screened a total of 68 individuals with 31 excluded as per exclusion criteria $(n=14)$, declining to commit to full participation $(n=13)$, or for other reasons $(n=4)$. Thirtyseven individuals were randomized to either RT $(n=20)$ or AT $(n=17)$. Figure 2 represents a Consolidated Standards of Reporting Trials (CONSORT) diagram. Of the 20 individuals randomized to RT, 18 completed this intervention. One individual was removed from the study after failing to comply with the medical staff's request to address a non-study related medical condition. Another individual was dismissed after experiencing recurring anxiety episodes in the robotic device. Two individuals failed to complete the AT treatment. One individual completed 24 aquatic exercise sessions before experiencing a non-study related ankle fracture. Another AT participant was withdrawn due to health and bowel management issues. One individual in the RT group was not included in the ergometry final analysis because he could not complete the arm ergometry test secondary to upper extremity spasticity. Except for the previous participants who dropped out for medical reasons, all individuals completed the protocol with no drop outs due to the exercise intensity or demand. Ultimately, participants completed $97 \%$ of the exercise interventions, namely 36 sessions under each exercise condition with $20 \%$ of the sessions rescheduled to complete the protocol prescribed exercise visits. 


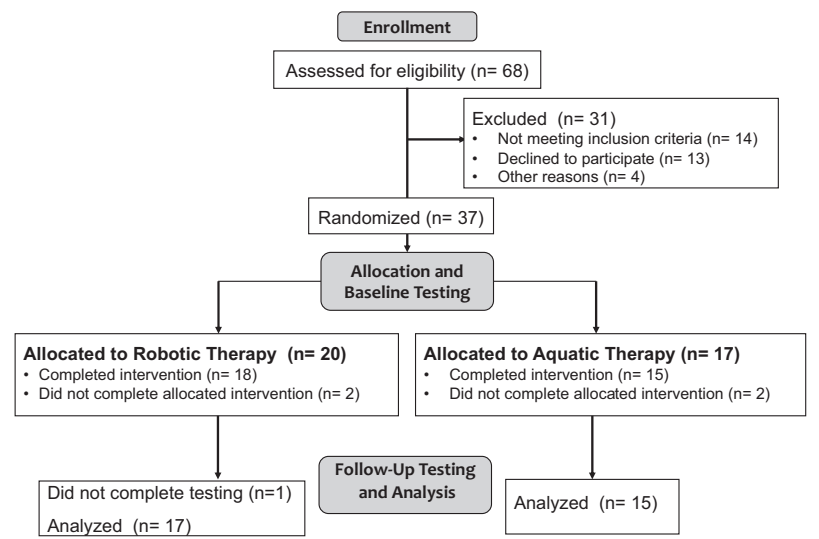

Fig. 2 CONSORT diagram of enrollment of participants into the study

Table 2 Baseline demographics

\begin{tabular}{lll}
\hline & Aquatic $(n=15)$ & Robotic $(n=18)$ \\
\hline Age (yrs) & $46.9 \pm 9.9$ & $45.4 \pm 12.9$ \\
Body weight $(\mathrm{kg})$ & $87.7 \pm 20.7$ & $79.33 \pm 20.2$ \\
Time since injury (yrs) & $12.2 \pm 12.6$ & $6.6 \pm 4.3$ \\
Tetraplegics & $11(73 \%)$ & $12(67 \%)$ \\
Paraplegics & $4(27 \%)$ & $6(33 \%)$ \\
WISCI (0-20) & $9.5 \pm 7.6$ & $11.7 \pm 6.5$ \\
LEMS (0-50) & $31.2 \pm 11.9$ & $32.4 \pm 10.7$ \\
UEMS (0-50) & $42.9 \pm 8.0$ & $41.7 \pm 9.7$ \\
Community ambulation & $10(67 \%)$ & $16(83 \%)$ \\
\hline
\end{tabular}

Participants completing intervention are included

Values are mean \pm standard deviation for continuous variables or percentages for categorical variables

Yrs years, $k g$ kilogram, WISCI Walking Index for Spinal Cord Injury, LEMS lower extremity motor score, UEMS upper extremity motor score

\section{Baseline demographics}

Table 2 displays baseline demographic data for the two groups, including all enrollees including the following: age, body weight, time since injury (TSI), WISCI-III score, LEMS, UEMS, and arm ergometry peak $\mathrm{VO}_{2}$.

\section{Three-month intervention results}

A summary of peak $\mathrm{VO}_{2}$ results is provided in Table 3. More detailed exercise data are provided several supplemental files (Supplementary Tables 1, 2, and 3 and Supplementary Figures 1 and 2). Neither aquatic exercise nor robotic exercise produced statistical improvement in peak $\mathrm{VO}_{2}$ when measured by arm cycle ergometry. However, with robotic intervention and using robotic peak $\mathrm{VO}_{2}$ assessments, peak $\mathrm{VO}_{2}$ did improve significantly (2.07 


\begin{tabular}{|c|c|c|c|c|c|}
\hline \multirow{4}{*}{ 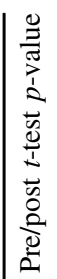 } & \multirow{4}{*}{ 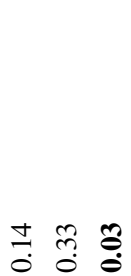 } & \multirow{3}{*}{ 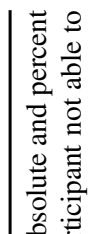 } & \multicolumn{3}{|c|}{$\begin{array}{l}\text { Table } 4 \text { Responders vs. non-responders (as defined by a } 10 \% \text { increase } \\
\text { in peak } \mathrm{VO}_{2} \text { ) across the 3-month exercise intervention }\end{array}$} \\
\hline & & & $\begin{array}{l}\text { A: Aquatic group (Arm } \\
\text { Ergometer } \mathrm{VO}_{2} \text { ) }\end{array}$ & $\begin{array}{l}\text { Responders } \\
(n=6)\end{array}$ & $\begin{array}{l}\text { Non-responders } \\
(n=9)\end{array}$ \\
\hline & & & Age (yrs) & $44.3 \pm 10.7$ & $48.6 \pm 8.3$ \\
\hline & & 光 & Time since injury (yrs) & $4.0 \pm 2.9$ & $17.7 \pm 12.9$ \\
\hline 2 & & ర్ & Weight (kg) & $77.3 \pm 21.6$ & $94.5 \pm 15.4$ \\
\hline 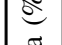 & $0^{0} \delta^{\circ}$ & $\frac{7}{2} 9$ & LEMS (0-50) & $35.2 \pm 11.6$ & $28.6 \pm 10.6$ \\
\hline$\ddot{\ddot{\nu}}$ & $\vec{\infty} \hat{0} \dot{ \pm}$ & 竞吾 & Baseline $\mathrm{VO}_{2}(\mathrm{ml} / \mathrm{kg} / \mathrm{min})$ & $13.1 \pm 3.2$ & $13.4 \pm 2.8$ \\
\hline ə & & 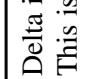 & $\begin{array}{l}\text { B: Robotic group (Arm } \\
\text { Ergometer } \mathrm{VO}_{2} \text { ) }\end{array}$ & $\begin{array}{l}\text { Responders } \\
(n=2)\end{array}$ & $\begin{array}{l}\text { Non-responders } \\
(n=15)\end{array}$ \\
\hline$\frac{7}{60}$ & & ฮี & Age (yrs) & $50.0 \pm 11.0$ & $46.0 \pm 12.1$ \\
\hline छ్ & &.$\frac{\vec{\pi}}{2} \frac{\pi}{\pi}$ & Time since injury (yrs) & $4.5 \pm 1.5$ & $6.9 \pm 4.5$ \\
\hline 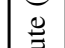 & & 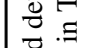 & Weight $(\mathrm{kg})$ & $80.9 \pm 8.2$ & $79.0 \pm 21.3$ \\
\hline 吾 & & 苛 & LEMS $(0-50)$ & $27.5 \pm 6.5$ & $33.0 \pm 11.0$ \\
\hline त्ञ & $\stackrel{\infty}{a} \hat{\imath}$ & 㯊 & Baseline $\mathrm{VO}_{2}(\mathrm{ml} / \mathrm{kg} / \mathrm{min})$ & $8.9 \pm 0.8$ & $17.5 \pm 4.7$ \\
\hline$\overline{\mathrm{Q}}$ & $\dot{0} \dot{i} i$ & 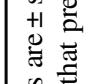 & $\begin{array}{l}\text { C: Robotic group (Robotic } \\
\text { Treadmill } \mathrm{VO}_{2} \text { ) }\end{array}$ & $\begin{array}{l}\text { Responders } \\
(n=11)\end{array}$ & $\begin{array}{l}\text { Non-responders } \\
(n=6)\end{array}$ \\
\hline : & & ฮี & Age (yrs) & $44.8 \pm 14.5$ & $45.2 \pm 8.9$ \\
\hline 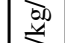 & & 己ृ & Time since injury (yrs) & $5.4 \pm 2.8$ & $7.2 \pm 4.5$ \\
\hline$\Xi$ & & है & Weight (kg) & $78.7 \pm 21.9$ & $84.3 \pm 12.6$ \\
\hline ᄋ & & $\vec{\exists}$ & LEMS (0-50) & $33.6 \pm 11.4$ & $31.7 \pm 8.7$ \\
\hline$\frac{\vec{c}}{\sigma}$ & $\dot{m} \vec{n} \quad 0$. & $\stackrel{\circ}{>}$ & Baseline $\mathrm{VO}_{2}(\mathrm{ml} / \mathrm{kg} / \mathrm{min})$ & $14.5 \pm 5.1$ & $15.6 \pm 1.2$ \\
\hline
\end{tabular}

Data are presented in three sections: $\mathrm{A}$ and $\mathrm{B}$-arm ergometry peak $\mathrm{VO}_{2}$ results for Aquatic and Robotic groups, respectively, and Crobotic treadmill peak $\mathrm{VO}_{2}$ results for Robotic group

Yrs years, $\mathrm{kg}$ kilograms, $\mathrm{ml}$ mililiters, LEMS lower extremity motor score

$\mathrm{ml} / \mathrm{kg} / \mathrm{min}, 14.7 \%, p=0.03, n=17)$. The effects of AT and RT on the primary outcome measure, namely arm ergometry measured peak $\mathrm{VO}_{2}$, were compared using a univariate ANOVA with the arm cycle ergometry being the consistent dependent variable. Even though it did not reach statistical significance $(p=0.063)$, AT provided a larger arm cycle ergometry peak $\mathrm{VO}_{2}$ change than RT.

\section{Responders vs. non-responders analysis}

To determine whether participant characteristics existed to identify a positive response to either of these two interventions, we assessed responder and non-responder groups. We defined a "responder" as a participant who exhibited a $10 \%$ or greater peak $\mathrm{VO}_{2}$ change. This definition was based on our prior work in this population [6], as well as previously published literature in a stroke survivor population [24] that demonstrated a reduced cardiorespiratory risk with $10 \%$ change. A responder vs. non-responder comparison produced no apparent statistically significant demographic factors predicting greater cardiorespiratory response (Table 4). It should be noted, however, that when robotic 
treadmill peak $\mathrm{VO}_{2}$ was compared to the arm ergometry peak $\mathrm{VO}_{2}$, the responder/non-responder ratio changed considerably (from 2:15 to 11:6). We repeated the responder/non-responder comparison using percentage cutoffs ranging from 5 to $9 \%$ improvement in peak $\mathrm{VO}_{2}$ based on our overall average improvement in arm ergometry $\mathrm{VO}_{2}$ and were unable to identify demographic factors that identified who would respond. Nonetheless, there were some trends noted, namely responders tended to be younger, more recently injured, and lighter weight than non-responders in the aquatic therapy group, and in the robotic therapy group when measurements were made with robotic ergometry.

\section{Discussion}

Aquatic exercise in peak arm ergometry $\mathrm{VO}_{2}$ demonstrated an average improvement of $8.1 \%$ with a wide variance. Our previous robotic treadmill intervention was conducted at $80-85 \%$ of HRR, in contrast to the lower intensity (65$75 \%)$ of this current study. The lower intensity (65-75\% HRR) was perhaps sufficient to induce a change in peak $\mathrm{VO}_{2}$ during robotic exercise, but may be insufficient to stimulate a change in cardiorespiratory fitness detectable via arm ergometry. This lack of sensitivity suggests that arm ergometry testing may not be the best tool to detect change in cardiorespiratory fitness following non-arm ergometry training interventions. This is despite previous work identifying a moderate correlation $(r=0.87)$ between arm ergometry and robotic $\mathrm{VO}_{2}$ measurement, although that study was not interventional [17].

Arm ergometry is an accepted measurement tool for assessing cardiorespiratory fitness in individuals with gait disturbances limiting treadmill testing [25, 26]. It may not however, be the most sensitive technique. The sensitivity difference may be due to differing muscle group activation during both the exercise intervention and cardiorespiratory testing. With coached robotic treadmill training and testing, the same leg and possibly trunk musculature are being activated as much as possible. In the water, upper extremity, trunk and preserved lower extremity musculature are recruited and augmented by gravity reduction and other hydrodynamic principles such as hydrostatic pressure, viscosity, and turbulence. Cardiorespiratory testing using arm ergometry recruits primarily the upper extremity and partial trunk musculature. Even though peak $\mathrm{VO}_{2}$ measurement is intended to assess primarily cardiorespiratory fitness, the technique used relies on specific musculature, perhaps limiting test sensitivity when the exercise intervention and the testing paradigm are not muscularly matched (such as when using arm ergometry to measure outcomes in robotic treadmill exercised individuals). This is particularly true since we included both paraplegic and tetraplegic individuals in the protocol, and impaired upper extremity function may have limited arm ergometry ability and performance in those with tetraplegia.

This study reproduced our previous findings that robotic treadmill therapy three times per week over 12 weeks improves cardiorespiratory fitness as measured by robotic treadmill peak $\mathrm{VO}_{2}$ measurements. The robotic treadmill peak $\mathrm{VO}_{2}$ improvement in this study across robotic randomized intervention was $14.7 \%$, similar to the $12.3 \%$ improvement reported in our prior single intervention protocol [6].

Specificity of intervention-test is reported with non-SCI participants. Pogliaghi et al. [27]. measured arm and leg cycling peak $\mathrm{VO}_{2}$ responses in healthy older males randomized to a 12 -week arm or leg cycling intervention. Both groups displayed increases in peak $\mathrm{VO}_{2}$ responses postintervention regardless of the exercise test modality. However, significantly higher peak $\mathrm{VO}_{2}$ responses occurred in the test modality specific to the training intervention. Another study by Bhambhani et al. [28] showed significantly improved peak $\mathrm{VO}_{2}$ responses in individuals with SCI randomized to an 8-week leg or arm cycling program with improvements only demonstrated by the test modality that matched the intervention phase for the respective randomized groups. Similarly, in a study of participants with peripheral arterial disease randomized to either arm or leg crank exercise, the improvement in peak $\mathrm{VO}_{2}$ was more robust when tested by the matched (arm or leg ergometry) technique [29]. To accurately capture cardiorespiratory adaptations our results combined with previous findings and literature stress the importance in selecting testing assessments specific to the training intervention. Both the robotic and aquatic testing data show task specificity is an important factor to consider when choosing test modalities to assess cardiorespiratory outcome before and after training those with incomplete SCI. Additionally, it is important to consider which cardiorespiratory training modality is more available for a given client. Rehabilitation institutions with wellness programs may include body weight support cardiorespiratory training, although these devices are not ubiquitous. Aquatic exercise is more likely to be readily available in a community setting and under some circumstances (depending on individual impairment and water safety) may be performed in a group format.

At this time, no peak $\mathrm{VO}_{2}$ minimal clinically important difference is established for individuals with SCI [30, 31]. One aspect of the importance of improvement in peak $\mathrm{VO}_{2}$ in people with CMISCI rests in the potential for greater capability and endurance in activities of daily living (ADL) performance. A study of individuals with paraplegia showed that improvement in peak $\mathrm{VO}_{2}$ positively correlated with self-reported physical activity [32]. The other benefit 
of improving peak $\mathrm{VO}_{2}$ is the potential reduction in cardiorespiratory disease and coronary heart disease, as demonstrated in the able-bodied population [33].

\section{Study limitations}

A relatively small sample size limited the study, although given the intensity of the intervention, recruitment of 37 participants across the two centers represents one of the larger SCI randomized exercise trials. Because of the complexity and length of the study, compliance with the schedule, especially over winter months, was a challenge, with a rescheduling rate approaching $20 \%$. Both robotic treadmill training and aquatic intervention protocols were designed to allow therapists to customize training based on individual participant needs while meeting this set study exercise structure. Dosage of each intervention remained consistent within the designed protocol, but different activities were utilized to optimize individual interventions. For example, a participant requiring increased hip flexion practiced flexion activities while another participant with preserved hip extension performed other activities such as hip abduction with both individuals maintaining the prescribed dosage intensity and time. Equipoise was maintained and interventions reflected customized clinical care within the study protocol structure.

We would have preferred to collect peak $\mathrm{VO}_{2}$ test during aquatic therapy itself, but the necessary equipment was not available at the time of this study. Our laboratory is currently evaluating the reliability and validity of peak $\mathrm{VO}_{2}$ responses obtained during deep water aerobic activities in individuals with SCI. Preliminary results show reliable and valid peak $\mathrm{VO}_{2}$ under these conditions [34]. We additionally determined 65-75\% HRR was potentially insufficient to maximize the change in cardiorespiratory fitness. When physically possible for participants, we recommend 75-85\% HRR for all cardiorespiratory training occurring in deep water thereby facilitating a greater cardiorespiratory response [35].

Blinding of the testers performing the peak $\mathrm{VO}_{2}$ measurement did not occur at both sites given the study complexities and manpower needs. Potential bias may be one concern but with multiple concurrent participants testers were evaluating individuals receiving different interventions on a regular basis. Furthermore, the testers held no direct knowledge of the preintervention results at the time of the post intervention testing.

The heterogeneity of our study participants, even with well described inclusion criteria, creates issues in determining and assessing meaningful cardiorespiratory change. Our struggle to identify homogeneous subgroups who could optimally benefit from one intervention or the other is not novel in clinical rehabilitation research $[36,37]$.
To optimize best care, it would be helpful to understand not only the intensity, frequency, and specific intervention parameters but also the duration, or how many visits facilitate best care to improve cardiorespiratory and cardiovascular health. Another important area to investigate is the exercise parameters that will maintain the improved cardiorespiratory health for individuals with SCI. As mentioned previously the large fitness variance in the SCI population (due to many factors) creates difficulties with exact rehabilitative and wellness intervention dosage. Although exercise guidelines are available for individuals with SCI, further refinement is needed [38].

Aquatic and robotic treadmill therapies are can safely improve cardiorespiratory fitness in individuals with CMISCI. The cardiorespiratory effect of coached robotic treadmill therapy provided a significant improvement as measured by a peak $\mathrm{VO}_{2}$ robotic treadmill testing paradigm, but not with an arm ergometry paradigm. In order to optimize the sensitivity of testing, it is best to match the intervention with the testing approach. Although not everyone responded to aquatic or robotic exercise intervention, this dataset suggests that younger age, a more recent time since injury, and lower body weight may facilitate increased cardiorespiratory improvement.

\section{Data archiving}

Data generated and analyzed during this study are included in this published article and its supplementary information files. This study is registered under ClinicalTrials.gov Identifier: NCT01407354, and some of the summary data are available at that repository as well.

Acknowledgements We acknowledge the clinical expertize of therapists: Sara Frye, Naomi Price-Miller, Jean McQuaid, Marni Kallins, Neshella Braga, Michelle Daniels, Rosalyn Lobo, R. Sandy Alexander, Lauren McCollough, Angela Pihera, Michelle Deyton, and Lisa Ruger. We also appreciate the work of research coordinators Gertrude Morrison and John Perreault.

Funding This work was funded by the Department of Defense CDMRP Spinal Cord Injury Research Program Clinical Trial Award SC090147, FY 2009.

Author contributions PHG was responsible for co-study design, cosubmitting the application for funding, screening, and medical supervision of participants at one center, writing the initial draft of the manuscript, and submission of completed manuscript. WS was responsible for daily study execution, robotic treadmill training and collection of all $\mathrm{VO}_{2}$ data at one center, initial statistical evaluation of the collected data, and major editing of the manuscript. LVH was responsible for co-design of the robotic treadmill algorithm and training, regulatory management and daily oversight including robotic treadmill training and aquatic therapy, $\mathrm{VO}_{2}$ data collection, screening and recruitment of participants at the second center, and review of the manuscript. KT was responsible for co-study design, medical oversight 
at the second center, co-authorship of the original funding application, and review of the manuscript. WMS was responsible for statistical analysis of the overall study. PRG was responsible for co-study design, co-submitting the application for funding, daily oversight of the dual center study, the design of the aquatic intervention, direct supervision of the aquatic intervention at one center and indirect at second site, initial statistical assessment with WS, and critical partial writing and review of the manuscript.

\section{Compliance with ethical standards}

Conflict of interest The authors declare that they have no conflicts of interest.

Ethical approval We certify that all applicable institutional and governmental regulations concerning the ethical use of human volunteers were followed during the course of this research.

Publisher's note: Springer Nature remains neutral with regard to jurisdictional claims in published maps and institutional affiliations.

\section{References}

1. Noreau L, Shephard RJ, Simard C, Pare G, Pomerleau P. Relationship of impairment and functional ability to habitual activity and fitness following spinal cord injury. Int $\mathrm{J}$ Rehabil Res. 1993;16:265-75.

2. Bresnahan JJ, Farkas GJ, Clasey JL, Yates JW, Gater DR. Arm crank ergometry improves cardiovascular disease risk factors and community mobility independent of body composition in high motor complete spinal cord injury. J Spinal Cord Med. 2018;41:1-21.

3. Gorgey AS, Graham ZA, Bauman WA, Cardozo C, Gater DR. Abundance in proteins expressed after functional electrical stimulation cycling or arm cycling ergometry training in persons with chronic spinal cord injury. J Spinal Cord Med. 2017;40:439-48.

4. Kim DI, Lee H, Lee BS, Kim J, Jeon JY. Effects of a 6-week indoor hand-bike exercise program on health and fitness levels in people with spinal cord injury: A randomized controlled trial study. Arch Phys Med Rehabil. 2015;96:2033-40 e1.

5. Crameri RM, Cooper P, Sinclair PJ, Bryant G, Weston A. Effect of load during electrical stimulation training in spinal cord injury. Muscle Nerve. 2004;29:104-11.

6. Gorman PH, Scott W, York H, Theyagaraj M, Price-Miller N, McQuaid J, et al. Robotically assisted treadmill exercise training for improving peak fitness in chronic motor incomplete spinal cord injury: A randomized controlled trial. J Spinal Cord Med. 2016;39:32-44.

7. Hoekstra F, van Nunen MP, Gerrits KH, Stolwijk-Swuste JM, Crins MH, Janssen TW. Effect of robotic gait training on cardiorespiratory system in incomplete spinal cord injury. J Rehabil Res Dev. 2013;50:1411-22.

8. Phillips SM, Stewart BG, Mahoney DJ, Hicks AL, McCartney N, Tang JE, et al. Body-weight-support treadmill training improves blood glucose regulation in persons with incomplete spinal cord injury. J Appl Physiol (1985). 2004;97:716-24.

9. Carlson KF, Wilt TJ, Taylor BC, Goldish GD, Niewoehner CB, Shamliyan TA, et al. Effect of exercise on disorders of carbohydrate and lipid metabolism in adults with traumatic spinal cord injury: systematic review of the evidence. J Spinal Cord Med. 2009;32:361-78.

10. Dobkin B, Apple D, Barbeau H, Basso M, Behrman A, Deforge $\mathrm{D}$, et al. Weight-supported treadmill vs over-ground training for walking after acute incomplete SCI. Neurology. 2006;66:484-93.

11. Hicks AL, Adams MM, Martin Ginis K, Giangregorio L, Latimer A, Phillips SM, et al. Long-term body-weight-supported treadmill training and subsequent follow-up in persons with chronic SCI: effects on functional walking ability and measures of subjective well-being. Spinal Cord. 2005;43:291-8.

12. Lefeber N, Swinnen E, Kerckhofs E. The immediate effects of robot-assistance on energy consumption and cardiorespiratory load during walking compared to walking without robotassistance: a systematic review. Disabil Rehabil Assist Technol. 2017;12:657-71.

13. Jack LP, Allan DB, Hunt KJ. Cardiopulmonary exercise testing during body weight supported treadmill exercise in incomplete spinal cord injury: a feasibility study. Technol Health Care. 2009;17:13-23.

14. Kressler J, Nash MS, Burns PA, Field-Fote EC. Metabolic responses to 4 different body weight-supported locomotor training approaches in persons with incomplete spinal cord injury. Arch Phys Med Rehabil. 2013;94:1436-42.

15. Soyupek F, Savas S, Ozturk O, Ilgun E, Bircan A, Akkaya A. Effects of body weight supported treadmill training on cardiac and pulmonary functions in the patients with incomplete spinal cord injury. J Back Musculoskelet Rehabil. 2009;22:213-8.

16. Field-Fote EC, Roach KE. Influence of a locomotor training approach on walking speed and distance in people with chronic spinal cord injury: a randomized clinical trial. Phys Ther. 2011;91:48-60.

17. Gorman PH, Geigle PR, Chen K, York H, Scott W. Reliability and relatedness of peak $\mathrm{VO} 2$ assessments during body weight supported treadmill training and arm cycle ergometry in individuals with chronic motor incomplete spinal cord injury. Spinal Cord. 2014;52:287-91.

18. Giesecke CL. Aquatic rehabilitation of clients with spinal cord injury. In: R RG, M DM, Cole AJ, editors. Aquatic rehabilitation. Philadelphia: Lippincott-Raven; 1997. p. 127-50.

19. Brody LT, Geigle PR. Aquatic exercise for rehabilitation and training. Champaign, IL: Human Kinetics; 2009. p. 352.

20. Stevens SL, Caputo JL, Fuller DK, Morgan DW. Effects of underwater treadmill training on leg strength, balance, and walking performance in adults with incomplete spinal cord injury. $\mathbf{J}$ Spinal Cord Med. 2015;38:91-101.

21. Borg G. Ratings of perceived exertion and heart rates during short-term cycle exercise and their use in a new cycling strength test. Int J Sports Med. 1982;3:153-8.

22. Gulick D. Aquatic cardiopulmonary training in aquatic rehabilitation and training. Champaign, Illinois: Human Kinetics; 2009.

23. Gass GC, Watson J, Camp EM, Court HJ, McPherson LM, Redhead P. The effects of physical training on high level spinal lesion patients. Scand J Rehabil Med. 1980;12:61-5.

24. Macko RF, DeSouza CA, Tretter LD, Silver KH, Smith GV, Anderson PA, et al. Treadmill aerobic exercise training reduces the energy expenditure and cardiovascular demands of hemiparetic gait in chronic stroke patients. A preliminary report. Stroke. 1997;28:326-30.

25. Hasnan N, Ektas N, Tanhoffer AI, Tanhoffer R, Fornusek C, Middleton JW, et al. Exercise responses during functional electrical stimulation cycling in individuals with spinal cord injury. Med Sci Sports Exerc. 2013;45:1131-8.

26. Hopman MT, Dallmeijer AJ, Snoek G, van der Woude LH. The effect of training on cardiovascular responses to arm exercise in individuals with tetraplegia. Eur J Appl Physiol Occup Physiol. 1996;74:172-9.

27. Pogliaghi S, Terziotti P, Cevese A, Balestreri F, Schena F. Adaptations to endurance training in the healthy elderly: arm cranking versus leg cycling. Eur J Appl Physiol. 2006;97:723-31. 
28. Bhambhani YN, Eriksson P, Steadward RD. Reliability of peak physiological responses during wheelchair ergometry in persons with spinal cord injury. Arch Phys Med Rehabil. 1991;72:559-62.

29. Zwierska I, Walker RD, Choksy SA, Male JS, Pockley AG, Saxton JM. Upper- vs lower-limb aerobic exercise rehabilitation in patients with symptomatic peripheral arterial disease: a randomized controlled trial. J Vasc Surg. 2005;42:1122-30.

30. Wu X, Liu J, Tanadini LG, Lammertse DP, Blight AR, Kramer $\mathrm{JL}$, et al. Challenges for defining minimal clinically important difference (MCID) after spinal cord injury. Spinal Cord. 2015;53:84-91.

31. Scivoletto G, Tamburella F, Laurenza L, Molinari M. The spinal cord independence measure: how much change is clinically significant for spinal cord injury subjects. Disabil Rehabil. 2013;35:1808-13.

32. Manns PJ, McCubbin JA, Williams DP. Fitness, inflammation, and the metabolic syndrome in men with paraplegia. Arch Phys Med Rehabil. 2005;86:1176-81.

33. Kodama S, Saito K, Tanaka S, Maki M, Yachi Y, Asumi M, et al. Cardiorespiratory fitness as a quantitative predictor of all-cause mortality and cardiovascular events in healthy men and women: a meta-analysis. JAMA. 2009;301:2024-35.

34. Geigle P, Ogonowska-Slodownik A, Slodownik R, Gorman P, Scott W. Measuring peak volume of oxygen (peak VO2) in deep water for individuals with spinal cord injury. J Aquat Phys Ther. 2018;26:30-5.

35. Piotrowska-Całka E. The effects of a 24-week deep water aerobic training program on cardiovascular fitness. Biol Sport. 2010;27:95-8.

36. Tanadini LG, Steeves JD, Hothorn T, Abel R, Maier D, Schubert M, et al. Identifying homogeneous subgroups in neurological disorders: unbiased recursive partitioning in cervical complete spinal cord injury. Neurorehabil Neural Repair. 2014;28:507-15.

37. Wang YC, Hart DL, Stratford PW, Mioduski JE. Baseline dependency of minimal clinically important improvement. Phys Ther. 2011;91:675-88.

38. Nightingale TE, Metcalfe RS, Vollaard NB, Bilzon JL. Exercise guidelInes to promote cardiometabolic health in spinal cord injured humans: Time to raise the intensity? Arch Phys Med Rehabil. 2017;98:1693-704. 